\title{
SMR \\ Prenatal diagnosis of Chinese families with phenylketonuria
}

\author{
N. Liu' ${ }^{1}$ X.D. Kong1, D.H. Zhao' ${ }^{2}$, Q.H. Wu' ${ }^{1}$, X.L. Li' ${ }^{2}$, H.F. Guo ${ }^{4}$, L.X. Cui ${ }^{4}$, \\ M. Jiang ${ }^{1}$ and H.R. Shi ${ }^{3}$ \\ ${ }^{1}$ Prenatal Diagnosis Center, The First Affiliated Hospital of Zhengzhou University, \\ Zhengzhou, Henan, China \\ ${ }^{2}$ Neonatal Screening Center, The Third Affiliated Hospital of Zhengzhou University, \\ Zhengzhou, Henan, China \\ ${ }^{3}$ Department of Obstetrics and Gynecology, \\ The First Affiliated Hospital of Zhengzhou University, Zhengzhou, Henan, China \\ ${ }^{4}$ Neonatal Screening Center, Zhengzhou Maternity and Child Care Hospital, \\ Zhengzhou, Henan, China
}

Corresponding author: H.R. Shi

E-mail: shihuirong_I@163.com

Genet. Mol. Res. 14 (4): 14615-14628 (2015)

Received June 23, 2015

Accepted October 7, 2015

Published November 18, 2015

DOI http://dx.doi.org/10.4238/2015.November.18.25

ABSTRACT. The aim of this study is to investigate the ability to prenatally diagnose phenylketonuria (PKU) by using phenylalanine hydroxylase (PAH) gene mutation analysis combined with short tandem repeat (STR) linkage analysis in 118 fetuses from 112 Chinese families. Genomic DNA was extracted from the peripheral blood from members of 112 families and the exons and exon-intron boundaries of the $P A H$ gene were amplified by PCR. PCR products were analyzed by bi-directional Sanger sequencing and multiplex ligation-dependent probe amplification (MLPA). The three variable number of tandem repeat (VNTR) markers PAH-1, PAH-26, PAH32 were used in the prenatal diagnosis for the PKU families. We identified a spectrum of 63 different mutations, including 61 point mutations and indels, two large exon deletion mutations, and five novel mutations. A substantial proportion of mutant alleles were accounted for by p.R243Q (15.62\%), EX6-96AG (9.82\%), p.V399V (7.59\%), p.Y356X (6.70\%), and p.R413P 
(5.36\%). The same mutations were identified in 31 prenatally genotyped fetuses. We identified 58 fetuses that carried only one mutant allele and 29 fetuses that carried no mutations of $\mathrm{PAH}$ and were presumed normal. $P A H$ gene mutation analysis combined with STR linkage analysis can provide rapid and accurate prenatal diagnosis for PKU families.

Key words: Phenylketonuria; Phenylalanine hydroxylase gene; Mutation; Prenatal diagnosis

\section{INTRODUCTION}

Phenylketonuria (PKU; MIM 261600), the most common inherited disorder of amino acid metabolism, is autosomal recessive and caused by mutations of the phenylalanine hydroxylase (PAH) gene. The overall incidence of PKU in China is $1 / 11,000$ (Gu and Wang, 2004). Loss or absence of $\mathrm{PAH}$ activity results in increased concentrations of phenylalanine in the blood and toxic concentrations in the brain. Untreated phenylketonuria is associated with progressive intellectual impairment and can be associated with eczematous rash, autism, seizures, and motor deficits (Blau et al., 2010). Developmental problems, aberrant behavior, and psychiatric symptoms often become apparent as the child grows. Although PKU is a curable inherited disease and an early phenylalanine restricted diet results in $90 \%$ of patients having normal mental development, the expensive cost, unstable curative effect, and poor treatment adherence cause some children to suffer from the disease. Prenatal diagnosis is the most effective method to avoid PKU-caused complications. However, a prenatal diagnosis cannot be made from sampling the PAH enzyme in the amniotic fluid as the enzyme is only expressed by the liver (Woo et al., 1983). Therefore, prenatal diagnosis of PKU is feasible only by molecular studies. Cloned human $P A H$ gene allows prenatal diagnosis by DNA analysis to deduce the phenotype of the fetus. Currently there are two genotype assays. The first, an alternative approach to mutation analysis, is by linkage analysis of short tandem repeat (STR) polymorphic markers associated with the $P A H$ gene locus in intron 3 (Goltsov et al., 1993). This method is mainly used in early prenatal diagnosis but requires that the affected child is available and that a firm diagnosis of PKU has been established. Secondly, Sanger sequencing and multiplex ligation-dependent probe amplification (MLPA) based on PCR can be used. For our study, we used Sanger sequencing and MLPA for gene analysis combined with STR linkage analysis, thereby providing the prenatal diagnosis of 118 fetuses from 112 families.

\section{MATERIAL AND METHODS}

\section{Subjects and methods}

This study was performed at the FirstAffiliated Hospital of Zhengzhou University (Zhengzhou, China), was approved by the hospital's Ethics Committee, and was performed according to the principles of the Declaration of Helsinki. All subjects gave their written informed consent.

\section{Study subjects}

The 112 families analyzed in this study were recruited from the Genetic Counseling clinic of the Prenatal Diagnosis Center of the First Affiliated Hospital and the Third Affiliated Hospital 
of Zhengzhou University from January 2009 to March 2014. One couple was a consanguineous marriage (cousins). All the probands in the research had abnormal blood phenylalanine (Phe) concentrations $(>120 \mu \mathrm{M})$ and had differential diagnosis detected by BH4-loading test, excluding $\mathrm{BH} 4$ deficiency. Five probands were deceased, and thus, their parents were used in place. Five families had twins; thus, we tested 118 fetuses from 112 PKU families.

\section{Collection of samples and genomic DNA extraction}

Peripheral blood samples were collected from the patients and parents in 107 families and from the parents in the five families in which the proband was deceased. Ethylenediaminetetraacetic acid (EDTA) was used as an anti-coagulant. Transabdominal chorionic villus sampling, amniocentesis, and umbilical cord blood puncture were performed at different times during gestation to evaluate the at-risk fetus. Genomic DNA was isolated from each sample using a commercial kit (TIANamp DNA Kit, Tiangen Biotech, Beijing, China).

\section{PCR and DNA sequencing}

PCR primers were designed by Primer 5.0. The primers covered the sequences of all 13 coding exons of the PAH gene (Table 1). PCR was performed using the GeneAmp PCR System 9700 (Applied Biosystems, Foster City, CA). The reaction included 20 to 50 ng genomic DNA, 1 $\mu \mathrm{L}$ each primer, $13 \mu \mathrm{L} 2 \mathrm{X}$ Taq PCR MasterMix (contains dNTPs, Tris- $\mathrm{HCl}$, Taq polymerase, $\mathrm{KCl}$, and $\mathrm{MgCl}_{2}$ ), and $\mathrm{ddH}_{2} \mathrm{O}$ to a final volume of $25 \mu \mathrm{L}$ (Taq PCR Mastermix (KT201), Tiangen Biotech, Beijing, China). The PCR amplification was as follows: 5 min initial denaturation at $96^{\circ} \mathrm{C}$, followed by 35 cycles of denaturation for $30 \mathrm{sec}$ at $96^{\circ} \mathrm{C}$, annealing for $40 \mathrm{~s}$ at 58 to $64^{\circ} \mathrm{C}$ (depending on the primer), and extension for $1 \mathrm{~min}$ at $72^{\circ} \mathrm{C}$ for $1 \mathrm{~min}$, followed by a final extension for $7 \mathrm{~min}$ at $72^{\circ} \mathrm{C}$. PCR products were evaluated by $1 \%$ agarose gel electrophoresis.

\begin{tabular}{|c|c|c|}
\hline Exon & Primer sequence $\left(5^{\prime} \rightarrow 3^{\prime}\right)$ & Length of PCR products(bp) \\
\hline$\overline{E 1}$ & $\begin{array}{l}\text { F: ATTTGAAGACCACTGGCCAAA } \\
\text { R: GAGTCCCGGAAGTGCCTAAAC }\end{array}$ & 656 \\
\hline E2 & $\begin{array}{l}\text { F: TTGCTTTGTCCATGGAGGTTT } \\
\text { R: ACAGGATCTGGAACAGGCAGA }\end{array}$ & 251 \\
\hline E3 & $\begin{array}{l}\text { F: TGTGAACTAACTGCCCCACCT } \\
\text { R: TTGCTGTTATTTTGCCCAAGC }\end{array}$ & 495 \\
\hline E4 & $\begin{array}{l}\text { F: GGGATCCCCACTTCTGATCTC } \\
\text { R: AACAACTCTGCCAACTGCAGG }\end{array}$ & 505 \\
\hline E5 & $\begin{array}{l}\text { F: CCCCCATTCAAAGCATTCATA } \\
\text { R: CATTCAAGATTTCAGGCCAGC }\end{array}$ & 543 \\
\hline E6 & $\begin{array}{l}\text { F: CCCTTTCATGTGGGAAATCAA } \\
\text { R: GTGCTTGTAGGAATGCATGCA }\end{array}$ & 481 \\
\hline E7 & $\begin{array}{l}\text { F: ATGTCCCTGGGCAGTTATGTG } \\
\text { R: TGAGAACAGGAACAAGTGGCA }\end{array}$ & 512 \\
\hline E8 & $\begin{array}{l}\text { F: GGGAGCATGTCCACAGGAATA } \\
\text { R: TATGATCCCACCTGAAATGGG }\end{array}$ & 470 \\
\hline E9 & $\begin{array}{l}\text { F: GGCCACCCATCACCTTTTTAT } \\
\text { R: GTAGCCCTTGAAAACCCTTGG }\end{array}$ & 387 \\
\hline E10 & $\begin{array}{l}\text { F: TCCCTTCATCCAGTCAAGGTG } \\
\text { R: ATTCCAAGGCTGACCTATGCA }\end{array}$ & 379 \\
\hline E11 & $\begin{array}{l}\text { F: CAGCATTTGGGCTGTGATGTA } \\
\text { R: CGTTCTCTGTTGGAAGGTTGG }\end{array}$ & 400 \\
\hline E12 & $\begin{array}{l}\text { F: ACCCTGCTCTAGGGAGGTGTC } \\
\text { R: CCTCTCCATCCCTTCTACGCT }\end{array}$ & 502 \\
\hline E13 & $\begin{array}{l}\text { F: AGCCCACTTATCCCCTAGTGC } \\
\text { R: ATTTGGGACCTGCTTCATTCA }\end{array}$ & 413 \\
\hline
\end{tabular}


The amplified products were purified with a cycle-pure kit (OMEGA, Bio-Tek, Doraville, GA) and bidirectionally sequenced using an ABI PRISM 3130-xI Genetic Analyzer (Applied Biosystems, Carlsbad, CA) to detect gene mutations. The resulting sequences were compared with the $P A H$ gene NCBI reference sequence (NG_008690.1). The variation sites of all patients' sequences were compared to those of the parents, to determine the origin of sequence variability. We identified the biological relationship in de novo mutation pedigrees with Promega PowerPlex $16 \mathrm{HS}$ system (Promega Corporation, Madison, WI, USA).

\section{MLPA analysis}

MLPA analysis was performed using the Salsa MLPA Kit P055 PAH (MRC-Holland, Amsterdam, The Netherlands) following DNA sequencing analysis to screen for deletions or duplications in PAH gene exons in seven hyperphenylalaninemics whose genotypes remained partially or completely undetermined. MLPA analysis was carried out by standard protocols. PCR products were identified and quantified using capillary electrophoresis on an ABI 3130-xI Genetic Analyzer with GeneScan software to size the PCR products and to obtain peak areas. For data analysis, the peak sizes and areas were transferred to an Excel file. For normalization, relative probe signals were calculated by dividing each measured peak area by the sum of all peak areas of that sample. The ratio of each relative probe signal from patients compared to control samples was then calculated. An exon deletion was classified as when the ratio was lower than 0.7 and the corresponding samples were subjected to a second MLPA analysis.

\section{STR analysis}

The three STR sites PAH-1, PAH-26, and PAH-32 were selected for linkage analysis of prenatal fetuses. The primers for PCR were designed according to the literature (Yao et al., 2007) and the $5^{\prime}$ end of the primer was tagged with fluorescein FAM. The PCR reaction was routine. PCR products $(5 \mu \mathrm{L})$ after degeneration were directly analyzed for fragment analysis on the ABI $3130-\mathrm{xl}$ Genetic Analyzer with the GeneMapper 4.2 software to size the PCR products.

\section{Nomenclature and sequence analysis}

The potential mutations were compared with the known disease-causing mutations deposited in disease databases, including the Human Gene Mutation Database (HGMD Professional 2014.3) and the mutation database for the human phenylalanine hydroxylase gene (http://www.pahdb. mcgill.ca/). We excluded single nucleotide polymorphisms (SNPs) by querying 1000 Genomes Data (http://www.1000genomes.org/), the dbSNP database, and the hapmap database. The novel mutations were named according to the Human Genomic Variation Society (HGVS).

\section{Bioinformatic analysis of the mutation sequences}

Evolutionary conservation of a non-synonymous variant was estimated with protein sequence alignment generated by ClustalW and compared with that presented by the Ensembl database. The functional consequences of the missense variants were predicted using Polymorphism Phenotyping (PolyPhen-2). Protein Variation Effect Analyzer (PROVEAN) scores, which predict whether a protein sequence variation affects protein function, were also determined. 


\section{Prenatal testing}

Fetal cells were obtained from transabdominal chorionic villi isolated during the first trimester, by amniocentesis performed during the second trimester, or by umbilical cord blood puncture during the third trimester. In 107 families, $P A H$ gene mutation analysis combined with linkage analysis method was used. For the other five families in which the probands were deceased, only $\mathrm{PAH}$ gene mutation analysis was used.

Maternal contamination from fetal samples and paternity was confirmed by using PowerPlex 16 HS System Kit to ensure the accuracy of linkage analysis, especially for the de novo family.

\section{Follow up}

Umbilical cord blood was collected at birth for genetic analysis. Heel blood from newborns was screened by Guthrie test. Additionally, urine organic acid analysis using tandem mass spectrometry was also used to confirm results. Developmental assessment of the newborn was performed with age.

\section{RESULTS}

\section{Mutations identification and analysis}

We identified 61 point mutations or indels and two large deletions in the coding region or adjacent intronic regions of the $P A H$ gene on 219 of 224 independent chromosomes. Additionally, we identified five novel mutations (Table 2).

We detected two allele mutations (including homozygotic mutations) in 107 families (including the five families for which the proband is deceased), but five families had only one allele mutation, furthermore, patients with $\mathrm{BH} 4$ cofactor deficiency were excluded.

We identified five novel mutations: p.D101N, c.463delC, p.Q172H, p.S250F, and p.L444F.

A substantial proportion of mutant exons and flanking introns $(80 \%)$ were within exon 7 (32.4\%), exon $11(16.4 \%)$, exon $6(14.6 \%)$, exon $12(9.6 \%)$, and exon $3(8.7 \%)$.

We detected the corresponding mutation sites of parents' samples and found one patient carried a de novo mutation; paternity testing identified the mutation as inherited.

\section{Sanger sequencing}

The spectrum was composed of 61 different point mutations and indels by Sanger sequencing. The majority was comprised of missense mutations (63.9\%), with ten splice-site mutations, six nonsense mutations, and six deletions; the latter three types are classified as null mutations. The 61 identified mutations were distributed across the PAH coding sequence. A substantial proportion of mutant alleles were accounted for by $p . R 243 Q(15.62 \%)$, EX6-96A $>$ G (9.82\%), p.V399V (7.59\%), p.Y356X (6.70\%), and p.R413P (5.36\%).

We identified a definite diagnosis by detecting two or three mutations on two alleles by using the method in 105 families; the remaining seven families only had one mutant allele.

\section{MLPA analysis}

We detected large deletions or duplications of the PAH gene by MLPA in seven patients, 
Table 2. Spectrum of $P A H$ gene mutations detected in the 112 families.

\begin{tabular}{|c|c|c|c|c|c|c|}
\hline No. & Trivial name (Protein effect) & Systematic name (DNA level) & Location & Characters of mutation & No. of alleles & Relative frequency (\%) \\
\hline & IVS1-3T>C & c. $61-3 \mathrm{~T}>\mathrm{C}$ & Intron 1 & Splice & 1 & 0.45 \\
\hline & c.131_133delAAG & p.E44_V45delinsV & Exon 2 & Deletion & 1 & 0.45 \\
\hline & $\mathrm{R} 53 \mathrm{H}$ & c. $158 \mathrm{G}>\mathrm{A}$ & Exon 2 & Missense & 1 & 0.45 \\
\hline & IVS2+5G>C & c. $168+5 G>C$ & Intron 2 & Splice & 1 & 0.45 \\
\hline & S70del & c.208-210delTCT & Exon 3 & Deletion & 3 & 1.34 \\
\hline & E79fx13 & c.222-225delGAAT & Exon 3 & Deletion & 3 & 1.34 \\
\hline & $\mathrm{D} 101 \mathrm{~N}^{*}$ & c. $301 \mathrm{G}>\mathrm{A}$ & Exon 3 & Missense & 1 & 0.45 \\
\hline & H107R & c. $320 A>G$ & Exon 3 & Missense & 2 & 0.89 \\
\hline & R111X & c. $331 \mathrm{C}>\mathrm{T}$ & Exon 3 & Nonsense & 10 & 4.46 \\
\hline & IVS4-1G>A & c. $442-1 \mathrm{G}>\mathrm{A}$ & Intron 4 & Splice & 11 & 4.91 \\
\hline & IVS4+1G>A & c. $441+1 \mathrm{G}>\mathrm{A}$ & Intron 4 & Splice & 1 & 0.45 \\
\hline & R155fsX40* & c.463delC & Exon 5 & Deletion & 1 & 0.45 \\
\hline & A156P & c. $466 \mathrm{G}>\mathrm{C}$ & Exon 5 & Missense & 1 & 0.45 \\
\hline & R158W & c. $472 \mathrm{C}>\mathrm{T}$ & Exon 5 & Missense & 3 & 1.34 \\
\hline & F161S & c. $482 \mathrm{~T}>\mathrm{C}$ & Exon 5 & Missense & 3 & 1.34 \\
\hline & $\mathrm{H} 170 \mathrm{Q}$ & c. $510 \mathrm{~T}>\mathrm{A}$ & Exon 5 & Missense & 2 & 0.89 \\
\hline & G171R & c. $511 \mathrm{G}>\mathrm{A}$ & Exon 6 & Missense & 1 & 0.45 \\
\hline & Q172H & c. $516 \mathrm{G}>\mathrm{T}$ & Exon 6 & Missense & 1 & 0.45 \\
\hline & R176X & c. $526 \mathrm{C}>\mathrm{T}$ & Exon 6 & Nonsense & 6 & 2.68 \\
\hline & W187X & c. $561 \mathrm{G}>\mathrm{A}$ & Exon 6 & Nonsense & 1 & 0.45 \\
\hline & $E X 6-96 A>G$ & c. $611 \mathrm{~A}>\mathrm{G}$ & Exon 6 & Splice & 22 & 9.82 \\
\hline & I224T & c. $671 \mathrm{~T}>\mathrm{C}$ & Exon 6 & Missense & 1 & 0.45 \\
\hline & Q232X & c. $694 \mathrm{C}>\mathrm{T}$ & Exon 7 & Nonsense & 1 & 0.45 \\
\hline & R241fsX5 & c.722delG & Exon 7 & Deletion & 1 & 0.45 \\
\hline & R241C & c.721C >T & Exon 7 & Missense & 8 & 3.57 \\
\hline & $\mathrm{R} 241 \mathrm{H}$ & c. $722 \mathrm{G}>\mathrm{A}$ & Exon 7 & Missense & 1 & 0.45 \\
\hline & L242F & c. $724 \mathrm{C}>\mathrm{T}$ & Exon 7 & Missense & 1 & 0.45 \\
\hline & R243Q & c.728G >A & Exon 7 & Missense & 35 & 15.62 \\
\hline & G247V & c. $740 \mathrm{G}>\mathrm{T}$ & Exon 7 & Missense & 5 & 2.23 \\
\hline & G247R & c. $739 \mathrm{G}>\mathrm{C}$ & Exon 7 & Missense & 1 & 0.45 \\
\hline & S250F & c. $749 \mathrm{C}>\mathrm{T}$ & Exon 7 & Missense & 1 & 0.45 \\
\hline & R252Q & c. $755 \mathrm{G}>\mathrm{A}$ & Exon 7 & Missense & 3 & 1.34 \\
\hline & R252W & c. $754 \mathrm{C}>\mathrm{T}$ & Exon 7 & Missense & 1 & 0.45 \\
\hline & G257V & c. $770 \mathrm{G}>\mathrm{T}$ & Exon 7 & Missense & 1 & 0.45 \\
\hline & R261Q & c. $782 \mathrm{G}>\mathrm{A}$ & Exon 7 & Missense & 6 & 2.68 \\
\hline & F263L & c. $787 \mathrm{C}>\mathrm{T}$ & Exon 7 & Missense & 1 & 0.45 \\
\hline & Q267E & c. $799 \mathrm{C}>\mathrm{G}$ & Exon 7 & Missense & 1 & 0.45 \\
\hline & P275L & c. $824 \mathrm{C}>\mathrm{T}$ & Exon 7 & Missense & 2 & 0.89 \\
\hline & E280K & c. $838 \mathrm{G}>\mathrm{A}$ & Exon 7 & Missense & 1 & 0.45 \\
\hline & IVS7+2T >A & c. $842+2 T>A$ & Intron 7 & Splice & 1 & 0.45 \\
\hline & IVS8-7A>G & c. $913-7 A>G$ & Intron 8 & Splice & 3 & 1.34 \\
\hline & А322T & c. $964 \mathrm{G}>\mathrm{A}$ & Exon 9 & Missense & 1 & 0.45 \\
\hline & I324N & c. $971 \mathrm{~T}>\mathrm{A}$ & Exon 10 & Missense & 1 & 0.45 \\
\hline & W326X & c. $977 \mathrm{G}>\mathrm{A}$ & Exon 10 & Nonsense & 1 & 0.45 \\
\hline & p.A342>Hfs & c.1024delG & Exon 10 & Deletion & 1 & 0.45 \\
\hline & G344S & c. $1030 \mathrm{G}>\mathrm{A}$ & Exon 10 & Missense & 1 & 0.45 \\
\hline & A345T & c. $1033 \mathrm{G}>\mathrm{A}$ & Exon 10 & Missense & 1 & 0.45 \\
\hline & IVS10-1G>T & c. $1066-1 \mathrm{G}>\mathrm{T}$ & Intron 10 & Splice & 1 & 0.45 \\
\hline & IVS10-11G>A & c. $1066-11 \mathrm{G}>A$ & Intron 10 & Splice & 1 & 0.45 \\
\hline & Y356X & c. $1068 \mathrm{C}>\mathrm{A}$ & Exon 11 & Nonsense & 15 & 6.70 \\
\hline & T380M & c. $1139 \mathrm{C}>\mathrm{T}$ & Exon 11 & Missense & 1 & 0.45 \\
\hline & V399V & c. $1197 \mathrm{~A}>\mathrm{T}$ & Exon 11 & Missense & 17 & 7.59 \\
\hline & R400T & c. $1199 \mathrm{G}>\mathrm{C}$ & Exon 11 & Missense & 2 & 0.89 \\
\hline & IVS11+2T>C & c. $1199+2 \mathrm{~T}>\mathrm{C}$ & Intron 11 & Splice & 1 & 0.45 \\
\hline & R408W & c. $1222 \mathrm{C}>\mathrm{T}$ & Exon 12 & Missense & 2 & 0.89 \\
\hline & R408Q & c. $1223 G>A$ & Exon 12 & Missense & 1 & 0.45 \\
\hline & R413P & c. $1238 G>C$ & Exon 12 & Missense & 12 & 5.36 \\
\hline & T418P & c. $1252 A>C$ & Exon 12 & Missense & 1 & 0.45 \\
\hline & Q419R & c. $1256 \mathrm{~A}>\mathrm{C}$ & Exon 12 & Missense & 1 & 0.45 \\
\hline & A434D & c. $1301 \mathrm{C}>\mathrm{A}$ & Exon 12 & Missense & 4 & 1.79 \\
\hline & L444F* & c.1330 C>T & Exon 13 & Missense & 1 & 0.45 \\
\hline & \multirow[t]{2}{*}{ Large deletion } & & Upstrea +E1 & Deletion & 1 & 0.45 \\
\hline & & & E5 & Deletion & 1 & 0.45 \\
\hline & Detected & & & & 219 & 97.77 \\
\hline & Unknown & & & & 5 & 2.23 \\
\hline & Total & & & & 224 & 100 \\
\hline
\end{tabular}


which had only one or no mutation in the PAH gene based on the Sanger sequencing. In two of the seven patients, we detected exon deletions. We found a deletion involving a 5' UTR and exon 1 in a single patient and a deletion of exon 5 in another patient (Figure 1).

A

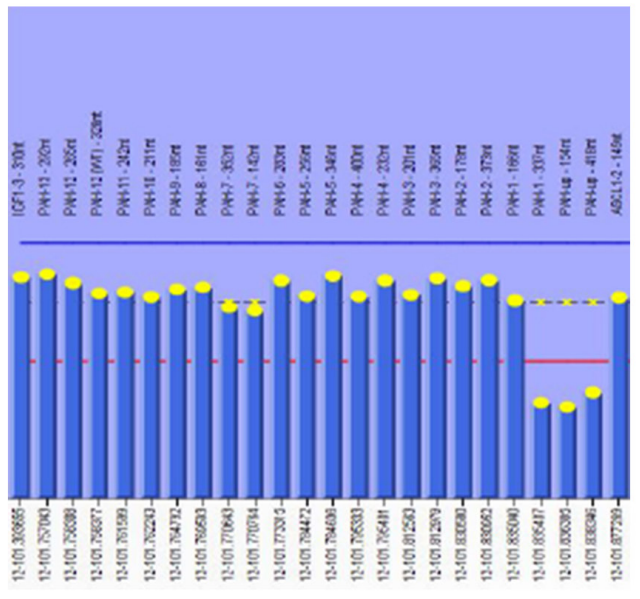

B

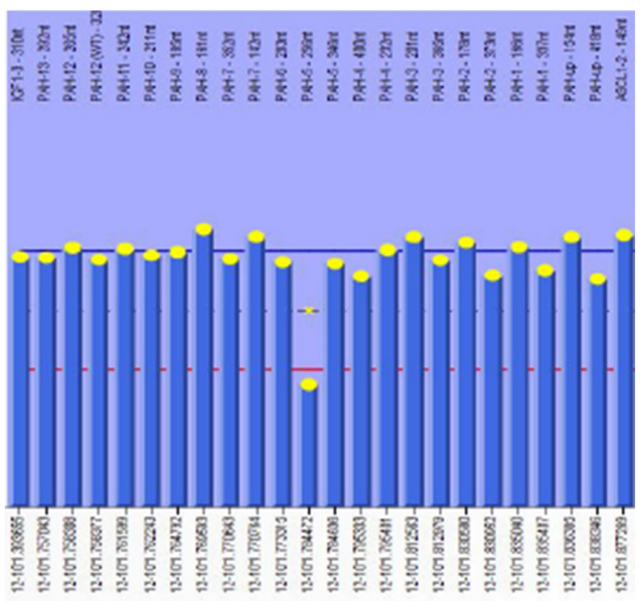

Figure 1. Multiplex ligation-dependent probe amplification analysis. The $\mathrm{X}$-axis of this bar diagram displays specific fragments, and the $\mathrm{Y}$-axis shows normalized ratios. Charts $\mathrm{A}$ and $\mathrm{B}$ respectively shows heterozygosity for a large deletion of $5^{\prime} U T R$ and exon 1 , exon 5 .

\section{STR analysis}

The three STR markers PAH-1, PAH-26, and PAH-32 had distinct heterozygosity, of which PAH-1 detected nine alleles $(254,250,246,242,238,234,232,230,226), \mathrm{PAH}-26$ detected six alleles $(223,221,219,217,215,213)$, and PAH-32 detected five alleles $(148,146,144,142$, 138). For 94 families $(79.7 \%)$, STR analysis could provide effective information; for 19 families, it could only provide exclusive information (16.1\%). For the remaining five families, no diagnostic information was provided by STR.

\section{Results of bioinformatic analysis of novel sequence variations}

The amino acid sequences of PAH protein in human, chimpanzee, sheep, mouse, and chicken were compared and analyzed. We found that the four novel missense variants sites of the PAH gene are highly conserved in all species (Figure 2). A combination of PROVEAN prediction and PolyPhen-2 showed that the majority of the novel sequence variations may be deleterious mutations rather than polymorphisms (Table 3 ).

\section{Prenatal diagnosis}

For 107 families, the prenatal fetus affected by PKU were detected by PAH gene mutation analysis combined with STR linkage analysis methods. For the remaining five families, we used only $P A H$ gene mutation analysis, as the proband is deceased. 


\begin{tabular}{|c|c|}
\hline Sheep & NLTHIESRPSRLRKDEYEFFTNLDQRSVPALAN I \\
\hline Mouse & NLTHIESRPSRLNKDEYEFFTYLDKRSKPVLGS \\
\hline Human & NLTHIESRPSRLKKDEYEFFTHLDKRSLPALTN \\
\hline Chicken & NLTHIESRPSRLKKDEYEFFTHLDKRSLPAL T \\
\hline Chimpanzee & $\begin{array}{l}\text { NLTHIESRPSRLKKDEYEFFTHLDKRSLPALTI } \\
* * * * * * * * * * * * . * * * * * * * * * * ; * * * . * \text {. }\end{array}$ \\
\hline Sheep & FPRT IQELDNFANQILSYGAELDADHPGFKDF \\
\hline Mouse & FPRT IQELDRFANQILSYGAELDADHPGFKDPV \\
\hline Human & FPRT IQELDRFANQILSYGAELDADHPGFKDP \\
\hline Chicken & FPRT IQELDRF ANQILSYGAELDADHPGFKDI \\
\hline Chimpanzee & $\begin{array}{l}\text { FPRTIQELDRFANQILSYGAELDADHPGFKDP } \\
* * * * * * * * * . * * * * * * * * * * * * * * * * * * * * * *\end{array}$ \\
\hline Sheep & RLRPVAGLISSRDFLGGLAFRVFHCTQYIRH \\
\hline Mouse & RLRPVAGLLSSRDFLGCLAFRVFHCTQYIRHG \\
\hline Human & RLRPVAGLLSSRDFLGGLAFRVFHCTQYIRHG \\
\hline Chicken & RLRPVAGLLSSSRDLGGLAFRVFHCTQYIRHG \\
\hline Chimpanzee & $\begin{array}{l}\text { RLRPVAGLLSSRDFLGCLAFRVFHCTQYIRHG } \\
* * * * * * * * * * * * * * * * * * * * * * * * * * * * * * * * * *\end{array}$ \\
\hline Sheep & IEVLDNTQQLKILADSISSEVE ILCSALQKLK- \\
\hline Mouse & VEVLDNTQQLKILADSINSEVGILCHALQKIKS \\
\hline Human & IEVLDNTQQLKILADSINSE IGHLCSALQK IK- \\
\hline Chicken & IEVLDNTQQLKILADSINSE IGHLCSALQKIK- \\
\hline Chimpanzee & $\begin{array}{l}\text { IEVLDNTQQLKILADSINSEIGILCSALQKIK- } \\
: * * * * * * * * * * * * * * * * . * *: ; * * * * * * *: *\end{array}$ \\
\hline
\end{tabular}

Figure 2. Genetic conservation of the six novel missense mutations. The amino acid sequence of the six codon position D101, Q172, S250, L444 (bounding box) in different species.

\begin{tabular}{|c|c|c|c|c|c|}
\hline \multirow[t]{5}{*}{ NO. } & Mutation type & Trivial name (Protein effect) & Systematic name (DNA level) & $\begin{array}{c}\text { In silico analysis (PolyPhen-2) } \\
\text { HumDiv Score }\end{array}$ & Classification \\
\hline & & D101N & c. $301 \mathrm{G}>\mathrm{A}$ & 0.000 & Benign \\
\hline & & Q172H & c. $516 \mathrm{G}>\mathrm{T}$ & 0.705 & Damaging \\
\hline & & S250F & c.749C $>\mathrm{T}$ & 1.000 & Damaging \\
\hline & & L444F & c. $1330 \mathrm{C}>\mathrm{T}$ & 0.999 & Damaging \\
\hline
\end{tabular}

Of the 118 fetuses, 112 were definitively diagnosed by $P A H$ gene mutation analysis (diagnostic rate of $91.5 \%$ ). By STR linkage analysis, 94 of 118 fetuses were definitively diagnosed (diagnostic rate of $79.7 \%$ ). In addition, the five families carrying one mutant allele were diagnosed using gene mutation analysis and STR linkage analysis.

Among the 118 prenatal genotype fetuses, 31 carried compound heterozygous alleles. Analysis of the aborted tissue confirmed that these fetuses carried two mutant alleles. We identified 58 fetuses as heterozygous, carrying one mutant allele, and 29 fetuses did not carry any detectable mutant allele (Table 4). 
Table 4. Prenatal genetic diagnosis in 112 families with PKU.

\begin{tabular}{|c|c|c|c|c|c|c|c|}
\hline \multirow[t]{2}{*}{ Families } & \multicolumn{2}{|c|}{ Proband genotype } & \multicolumn{2}{|c|}{ Parental genotype } & \multicolumn{2}{|c|}{ Fetal genotype } & \multirow{2}{*}{$\begin{array}{l}\text { Fetal pregnancy } \\
\text { outcome }\end{array}$} \\
\hline & Mutation 1 & Mutation 2 & Father source & Mother sources & Mutation 1 & Mutation 2 & \\
\hline 1 & $\mathrm{R} 261 \mathrm{Q}$ & R413P & R413P & $\mathrm{R} 261 \mathrm{Q}$ & $\mathrm{R} 261 \mathrm{Q}$ & R413P & Abortion \\
\hline 2 & Y356X & & Y356X & & Y356X & & Normal birth \\
\hline 3 & $E X 6-96 A>G$ & $R 243 Q$ & $E X 6-96 A>G$ & $R 243 Q$ & $E X 6-96 A>G$ & & Normal birth \\
\hline 4 & $\mathrm{R} 111 \mathrm{X}$ & $E X 6-96 A>G$ & $\mathrm{R} 111 \mathrm{X}$ & $E X 6-96 A>G$ & $\mathrm{R} 111 \mathrm{X}$ & & Normal birth \\
\hline 5 & IVS4-1G>A & G247V & IVS4-1G>A & G247V & & & Normal birth \\
\hline 6 & E79FX13 & $R 243 Q$ & $\mathrm{R} 243 \mathrm{Q}$ & E79FX13 & E79FX13 & & Normal birth \\
\hline 7 & $E X 6-96 A>G$ & $R 243 Q$ & $R 243 Q$ & $E X 6-96 A>G$ & $E X 6-96 A>G$ & & Normal birth \\
\hline 8 & $\mathrm{R} 243 \mathrm{Q}$ & V399V & $\mathrm{R} 243 \mathrm{Q}$ & V399V & & & Normal birth \\
\hline 9 & W326X & Y356X & W326X & Y356X & W326X & & Normal birth \\
\hline 10 & R252W & Y356X & Y356X & $\mathrm{R} 252 \mathrm{~W}$ & $\mathrm{R} 252 \mathrm{~W}$ & Y356X & Abortion \\
\hline 11 & $E X 6-96 A>G$ & IVS10-11G>A & $E X 6-96 A>G$ & IVS10-11G>A & $E X 6-96 A>G$ & IVS10-11G>A & Abortion \\
\hline 12 & IVS1-3T $>C$ & $\mathrm{R} 243 \mathrm{Q}$ & $\mathrm{R} 243 \mathrm{Q}$ & IVS1-3T>C & $\mathrm{R} 243 \mathrm{Q}$ & & Normal birth \\
\hline 13 & IVS4-1G>A & Y356X & IVS4-1G>A & Y356X & IVS4-1G>A & & Normal birth \\
\hline 14 & $\mathrm{R} 261 \mathrm{Q}$ & R413P & R413P & $\mathrm{R} 261 \mathrm{Q}$ & $\mathrm{R} 261 \mathrm{Q}$ & R413P & Abortion \\
\hline 15 & $\mathrm{R} 111 \mathrm{X}$ & $E X 6-96 A>G$ & R111X & $E X 6-96 A>G$ & & & Normal birth \\
\hline 16 & Y356X & A434D & Y356X & A434D & Y356X & & Normal birth \\
\hline 17 & & & $E X 6-96 A>G$ & $\mathrm{R} 243 \mathrm{Q}$ & $\mathrm{R} 243 \mathrm{Q}$ & & Normal birth \\
\hline 18 & Y356X & Y356X & Y356X & Y356X & Y356X & Y356X & Abortion \\
\hline 19 & G257V & V399V & G257V & V399V & G257V & & Normal birth \\
\hline 20 & S70del & $R 243 Q$ & $\mathrm{R} 243 \mathrm{Q}$ & S70del & S70del & R243Q & Abortion \\
\hline 21 & R111X & $R 243 Q$ & R243Q & $\mathrm{R} 111 \mathrm{X}$ & $\mathrm{R} 111 \mathrm{X}$ & & Normal birth \\
\hline 22 & R243Q & $R 243 Q$ & R243Q & $\mathrm{R} 243 \mathrm{Q}$ & & & Normal birth \\
\hline 23 & $\mathrm{R} 243 \mathrm{Q}$ & V399V & V399V & $\mathrm{R} 243 \mathrm{Q}$ & $\mathrm{R} 243 \mathrm{Q}$ & V399V & Abortion \\
\hline 24 & $\mathrm{R} 111 \mathrm{X}$ & R413P & $\mathrm{R} 111 \mathrm{X}$ & R413P & R413P & & Normal birth \\
\hline 25 & EX6-96A>G & V399V & $E X 6-96 A>G$ & V399V & $E X 6-96 A>G$ & & Normal birth \\
\hline 26 & & & R243Q & $\mathrm{R} 243 \mathrm{Q}$ & R243Q & & Normal birth \\
\hline 27 & $\mathrm{R} 243 \mathrm{Q}$ & $R 243 Q$ & $\mathrm{R} 243 \mathrm{Q}$ & $\mathrm{R} 243 \mathrm{Q}$ & & & Normal birth \\
\hline 28 & $R 243 Q$ & $\mathrm{R} 261 \mathrm{Q}$ & $\mathrm{R} 261 \mathrm{Q}$ & $\mathrm{R} 243 \mathrm{Q}$ & $\mathrm{R} 243 \mathrm{Q}$ & $\mathrm{R} 261 \mathrm{Q}$ & Abortion \\
\hline 29 & $E X 6-96 A>G$ & R261Q & R261Q & $E X 6-96 A>G$ & $E X 6-96 A>G$ & R261Q & Abortion \\
\hline 30 & IVS4-1G>A & R400T & $\mathrm{F} 263 \mathrm{~L}$ & R400T & F263L & R400T & Abortion \\
\hline 31 & IVS4-1G>A & c.722delG & IVS4-1G>A & c.722delG & c.722delG & & Normal birth \\
\hline 32 & G247V & & G247V & & G247V & & Normal birth \\
\hline 33 & IVS4-1G>A & R158W & R158W & IVS4-1G>A & IVS4-1G>A & R158W & Abortion \\
\hline 34 & Y356X & $\mathrm{R} 241 \mathrm{C}$ & Y356X & $\mathrm{R} 241 \mathrm{C}$ & Y356X & & Normal birth \\
\hline 35 & $\mathrm{R} 243 \mathrm{Q}$ & R243Q & R243Q & $\mathrm{R} 243 \mathrm{Q}$ & $\mathrm{R} 243 \mathrm{Q}$ & R243Q & Abortion \\
\hline 36 & $\mathrm{R} 252 \mathrm{Q}$ & $1324 \mathrm{~N}$ & $1324 \mathrm{~N}$ & R252Q & R252Q & $1324 \mathrm{~N}$ & Abortion \\
\hline 37 & $\mathrm{G} 247 \mathrm{~V}$ & IVS11+2T>C & $\mathrm{G} 247 \mathrm{~V}$ & IVS11+2T>C & IVS11+2T>C & & Normal birth \\
\hline 38 & & & $\mathrm{R} 243 \mathrm{Q}$ & & & & Normal birth \\
\hline 39 & $E X 6-96 A>G$ & $R 243 Q$ & $E X 6-96 A>G$ & $R 243 Q$ & $E X 6-96 A>G$ & $R 243 Q$ & Abortion \\
\hline 40 & F161S & $\mathrm{R} 243 \mathrm{Q}$ & $\mathrm{R} 243 \mathrm{Q}$ & F161S & F161S & & Normal birth \\
\hline 41 & $\mathrm{R} 413 \mathrm{P}$ & A434D & $\mathrm{R} 413 \mathrm{P}$ & A434D & $\mathrm{R} 413 \mathrm{P}$ & & Normal birth \\
\hline 42 & IVS2+5G $>C$ & G247R & IVS2+5G>C & G247R & & & Normal birth \\
\hline 43 & E79FX13 & IVS8-7A>G & IVS8-7A>G & E79FX13 & E79FX13 & & Normal birth \\
\hline 44 & EX6-96A $>G$ & V399V & $E X 6-96 A>G$ & V399V & & & Normal birth \\
\hline 45 & $E X 6-96 A>G$ & $\mathrm{R} 261 \mathrm{Q}$ & $\mathrm{R} 261 \mathrm{Q}$ & EX6-96A>G & $\mathrm{R} 261 \mathrm{Q}$ & & Normal birth \\
\hline 46 & R176X & $\mathrm{R} 243 \mathrm{Q}$ & $\mathrm{R} 243 \mathrm{Q}$ & $\mathrm{R} 176 \mathrm{X}$ & $\mathrm{R} 243 \mathrm{Q}$ & & Normal birth \\
\hline 47 & I224T & $R 243 Q$ & I224T & $\mathrm{R} 243 \mathrm{Q}$ & & & Normal birth \\
\hline 48 & IVS4-1G>A & R243Q & IVS4-1G>A & $\mathrm{R} 243 \mathrm{Q}$ & $\mathrm{R} 243 \mathrm{Q}$ & & Normal birth \\
\hline 49 & V399V & $\begin{array}{l}5 \text { ' end of exon } 1 \text { and } \\
\text { the second large } \\
\text { fragment } L O H\end{array}$ & V399V & $\begin{array}{l}5^{\prime} \text { end of exon } 1 \text { and } \\
\text { the second large } \\
\text { fragment } L O H\end{array}$ & $\begin{array}{l}5^{\prime} \text { end of exon } 1 \text { and } \\
\text { the second large } \\
\text { fragment } \mathrm{LOH}\end{array}$ & & Normal birth \\
\hline 50 & S70del & Y356X & S70del & Y356X & Y356X & & Normal birth \\
\hline 51 & $\mathrm{R} 252 \mathrm{Q}$ & R413P & $\mathrm{R} 252 \mathrm{Q}$ & $\mathrm{R} 413 \mathrm{P}$ & $\mathrm{R} 413 \mathrm{P}$ & & Normal birth \\
\hline 52 & F161S & V399V & F161S & V399V & F161S & V399V & Abortion \\
\hline 53 & $\mathrm{R} 243 \mathrm{Q}$ & R413P & R243Q & R413P & $\mathrm{R} 243 \mathrm{Q}$ & R413P & Abortion \\
\hline 54 & $\mathrm{R} 243 \mathrm{Q}$ & E280K & $\mathrm{R} 243 \mathrm{Q}$ & E280K & $\mathrm{R} 243 \mathrm{Q}$ & E280K & Abortion \\
\hline 55 & IVS4-1G>A & $E X 6-96 A>G$ & $E X 6-96 A>G$ & IVS4-1G>A & IVS4-1G>A & & Normal birth \\
\hline 56 & $\mathrm{H} 170 \mathrm{Q}$ & $E X 6-96 A>G$ & $\mathrm{H} 170 \mathrm{Q}$ & P275L & $\mathrm{H} 170 \mathrm{Q}$ & P275L & Abortion \\
\hline 57 & IVS4-1G>A & P275L & IVS4-1G>A & $E X 6-96 A>G$ & & & Normal birth \\
\hline 58 & Y356X & A434D & A434D & Y356X & & & Normal birth \\
\hline 59 & $E X 6-96 A>G$ & $R 243 Q$ & $E X 6-96 A>G$ & $\mathrm{R} 243 \mathrm{Q}$ & $E X 6-96 A>G$ & & Normal birth \\
\hline 60 & $\mathrm{~A} 156 \mathrm{P}$ & EX6-96A>G & $\mathrm{A} 156 \mathrm{P}$ & EX6-96A>G & $\mathrm{A} 156 \mathrm{P}$ & EX6-96A>G & Abortion \\
\hline 61 & & & Y356X & $\mathrm{R} 241 \mathrm{C}$ & $\mathrm{R} 241 \mathrm{C}$ & & Normal birth \\
\hline
\end{tabular}

Continued on next page 


\begin{tabular}{|c|c|c|c|c|c|c|c|}
\hline \multirow[t]{2}{*}{ Families } & \multicolumn{2}{|c|}{ Proband genotype } & \multicolumn{2}{|c|}{ Parental genotype } & \multicolumn{2}{|c|}{ Fetal genotype } & \multirow{2}{*}{$\begin{array}{l}\text { Fetal pregnancy } \\
\text { outcome }\end{array}$} \\
\hline & Mutation 1 & Mutation 2 & Father source & Mother sources & Mutation 1 & Mutation 2 & \\
\hline 62 & $E X 6-96 A>G$ & R241C & $E X 6-96 A>G$ & R241C & $E X 6-96 A>G$ & & Normal birth \\
\hline 63 & $\mathrm{R} 243 \mathrm{Q}$ & $\mathrm{G} 247 \mathrm{~V}$ & $\mathrm{R} 243 \mathrm{Q}$ & $\mathrm{G} 247 \mathrm{~V}$ & $\mathrm{R} 243 \mathrm{Q}$ & & Normal birth \\
\hline 64 & $E X 6-96 A>G$ & А345T & $E X 6-96 A>G$ & А345T & & & Normal birth \\
\hline 65 & V399V & R413P & R413P & V399V & V399V & & Normal birth \\
\hline 66 & $\mathrm{R} 243 \mathrm{Q}$ & & & $\mathrm{R} 243 \mathrm{Q}$ & $\mathrm{R} 243 \mathrm{Q}$ & & Normal birth \\
\hline 67 & EX6-96A>G & $\mathrm{R} 243 \mathrm{Q}$ & EX6-96A>G & $\mathrm{R} 243 \mathrm{Q}$ & $\mathrm{R} 243 \mathrm{Q}$ & & Normal birth \\
\hline 68 & $E \times 6-96 A>G$ & c.47-48delCT & c.47-48delCT & $E X 6-96 A>G$ & & & Normal birth \\
\hline 69 & R158W & A434D & A434D & R158W & & & Normal birth \\
\hline 70 & $\mathrm{R} 111 \mathrm{X}$ & Y356X & Y356X & $\mathrm{R} 111 \mathrm{X}$ & $\mathrm{R} 111 \mathrm{X}$ & & Normal birth \\
\hline 71 & $\mathrm{H} 170 \mathrm{Q}$ & $\mathrm{P} 275 \mathrm{~L}$ & $\mathrm{H} 170 \mathrm{Q}$ & $\mathrm{P} 275 \mathrm{~L}$ & $\mathrm{H} 170 \mathrm{Q}$ & P275L & Abortion \\
\hline 72 & F263L & R400T & F263L & R400T & & & Normal birth \\
\hline 73 & F161S & V399V & V399V & F161S & $\begin{array}{l}\text { F161S } \\
\text { V399V }\end{array}$ & V399V & $\begin{array}{l}\text { Abortion } \\
\text { Normal birth }\end{array}$ \\
\hline 74 & L242F & V399V & L242F & V399V & L242F & & Normal birth \\
\hline 75 & Q232X & $\mathrm{G} 247 \mathrm{~V}$ & $\mathrm{G} 247 \mathrm{~V}$ & Q232X & Q232X & & Normal birth \\
\hline 76 & & & R413P & $\mathrm{R} 243 \mathrm{Q}$ & R413P & & Normal birth \\
\hline 77 & R111X & V399V & V399V & $\mathrm{R} 111 \mathrm{X}$ & R111X & & Normal birth \\
\hline 78 & $R 243 Q$ & $\mathrm{R} 261 \mathrm{Q}$ & $\mathrm{R} 243 \mathrm{Q}$ & R261Q & $\mathrm{R} 243 \mathrm{Q}$ & $\mathrm{R} 261 \mathrm{Q}$ & Abortion \\
\hline 79 & R413P & R413P & R413P & R413P & R413P & R413P & Abortion \\
\hline 80 & Y356X & & & Y356X & & & Normal birth \\
\hline 81 & R408W & MLPA E5 LOH & MLPA E5 LOH & R408W & R408W & MLPA E5 LOH & Abortion \\
\hline 82 & IVS4+1G>A & EX6-96G $>A$ & IVS4+1G >A & EX6-96G>A & IVS4+1G>A & EX6-96G $>A$ & Abortion \\
\hline 83 & Y356X & V399V & Y356X & V399V & Y356X & & Normal birth \\
\hline 84 & W187X & V399V & V399V & W187X & & & Normal birth \\
\hline 85 & IVS7+2T>A & Y356X & IVS7+2T>A & Y356X & IVS7+2T>A & & Normal birth \\
\hline 86 & $\mathrm{D} 101 \mathrm{~N}^{*}$ & Q267E & $\mathrm{D} 101 \mathrm{~N}$ & Q267E & Q267E & & Pregnancy \\
\hline 87 & E79FX13 & IVS8-7A>G & IVS8-7A>G & E79FX13 & & & Pregnancy \\
\hline 88 & R111X & R413P & R111X & & R111X & & Normal birth \\
\hline 89 & R176X & $\mathrm{R} 241 \mathrm{C}$ & $\mathrm{R} 241 \mathrm{C}$ & $\mathrm{R} 176 \mathrm{X}$ & & & Pregnancy \\
\hline 90 & c. $463 \mathrm{delC}^{*}$ & $R 243 Q$ & $\mathrm{R} 243 \mathrm{Q}$ & c.463delC & $R 243 Q$ & & Pregnancy \\
\hline 91 & $\mathrm{H} 107 \mathrm{R}$ & R413P & $\mathrm{H} 107 \mathrm{R}$ & R413P & R413P & & Pregnancy \\
\hline 92 & R111X & R176X & R176X & $\mathrm{R} 111 \mathrm{X}$ & R176X & & Pregnancy \\
\hline 93 & $\mathrm{H} 107 \mathrm{R}$ & $\mathrm{R} 241 \mathrm{C}$ & H107R & $\mathrm{R} 241 \mathrm{C}$ & & & Pregnancy \\
\hline 94 & $\mathrm{R} 241 \mathrm{H}$ & L444F $^{*}$ & L444F & $\mathrm{R} 241 \mathrm{H}$ & $\mathrm{R} 241 \mathrm{H}$ & & Pregnancy \\
\hline 95 & IVS4-1G>A & R176X & IVS4-1G>A & R176X & IVS4-1G>A & R176X & Abortion \\
\hline 96 & V399V & V399V & V399V & V399V & & & Pregnancy \\
\hline 97 & $\mathrm{R} 241 \mathrm{C}$ & V399V & V399V & R241C & R241C & V399V & Abortion \\
\hline 98 & $\mathrm{R} 53 \mathrm{H}$ & $\mathrm{S}^{250 \mathrm{~F}^{*}}$ & $\mathrm{R} 53 \mathrm{H}$ & S250F & & & Pregnancy \\
\hline 99 & Q172H* & R408Q & Q172H & $\mathrm{R} 408 \mathrm{Q}$ & $\mathrm{R} 408 \mathrm{Q}$ & & Pregnancy \\
\hline 100 & $R 252 Q$ & T380M & T380M & $\mathrm{R} 252 \mathrm{Q}$ & $\mathrm{R} 252 \mathrm{Q}$ & & Pregnancy \\
\hline 101 & G344S & Q419R & G344S & Q419R & & & Pregnancy \\
\hline 102 & c.131-133delAAG & R408W & c. $131-133$ delAAG & R408W & c.131-133delAAG & R408W & Abortion \\
\hline 103 & R176X & A322T & R176X & A322T & A322T & & Pregnancy \\
\hline 104 & R111X & IVS8-7A>G & IVS8-7A>G & $\mathrm{R} 111 \mathrm{X}$ & $\mathrm{R} 111 \mathrm{X}$ & & Pregnancy \\
\hline 105 & $\mathrm{R} 111 \mathrm{X}$ & IVS10-1G>T & IVS10-1G>T & $\mathrm{R} 111 \mathrm{X}$ & & & Pregnancy \\
\hline 106 & R241C & $\mathrm{T} 418 \mathrm{P}$ & R241C & $\mathrm{T} 418 \mathrm{P}$ & R241C & & Pregnancy \\
\hline 107 & S70del & & & S70del & & & Pregnancy \\
\hline 108 & $R 243 Q$ & c.1024delG & $\mathrm{R} 243 \mathrm{Q}$ & c.1024delG & & & Pregnancy \\
\hline 109 & G171R & $E X 6-96 G>A$ & G171R & EX6-96G $>A$ & & & Pregnancy \\
\hline 110 & IVS4-1G>A & R158W & IVS4-1G>A & R158W & IVS4-1G>A & & Pregnancy \\
\hline 111 & $\mathrm{R} 241 \mathrm{C}$ & V399V & V399V & R241C & R241C & & Pregnancy \\
\hline 112 & IVS4-1G>A & R176X & IVS4-1G>A & R176X & IVS4-1G>A & R176X & Abortion \\
\hline 3 & EX6-96G $>A$ & $R 243 Q$ & $E X 6-96 G>A$ & $R 243 Q$ & EX6-96G >A & $R 243 Q$ & Abortion \\
\hline 35 & $\mathrm{R} 243 \mathrm{Q}$ & $\mathrm{R} 243 \mathrm{Q}$ & $\mathrm{R} 243 \mathrm{Q}$ & $\mathrm{R} 243 \mathrm{Q}$ & & & Normal birth \\
\hline 58 & Y356X & A434D & A434D & Y356X & A434D & & Normal birth \\
\hline 65 & V399V & R413P & R413P & V399V & V399V & R413P & Abortion \\
\hline 80 & Y356X & & & Y356X & & & Pregnancy \\
\hline
\end{tabular}

The fetus in family 88 only carried the mutant allele from the maternal genotype and had a de novo mutation after the paternity was confirmed by using PowerPlex 16 HS.

Family 73 had dizygotic twins identified by STR. Prenatal genotyping showed differences: one of the twins carried compound heterozygous alleles while the other only had one mutant allele. 
Following genetic counseling, the guardians chose selective reduction, which was undertook at gestational week 18.

\section{Genetic counseling and follow-up}

Following genetic counseling, 30 parents chose to terminate the pregnancy as their fetus carried compound heterozygous mutations. One couple chose to continue the pregnancy and the Phe serum concentration of the neonate was $1650 \mu \mathrm{M}(27.5 \mathrm{mg} / \mathrm{dL})$ by neonatal screening. The 87 carriers with normal fetuses continued their pregnancy, 64 had normal newborns passing neonatal screening, and the remaining 23 are still in pregnancy.

\section{DISCUSSION}

The PAH gene, located on human chromosome 12q23.2, was cloned by Woo et al. (1983). The gene contains 13 exons and the full-length of encoding region is $90 \mathrm{~kb}$, which encodes a monomer protein that is 452 amino acids. Four PAH proteins interact to form a tetramer, the functional unit of the enzyme, which interacts with four phenylalanine hydroxylase monomers. Previous studies have found over 766 different mutations in the PAH gene (HGMD Professional 2014.3). PAH gene analysis could provide an important basis for the $P A H$ gene spectrum representing different regions and races thereby allowing efficient gene diagnosis, genetic counseling, and prenatal diagnosis of PKU. Additionally, this tool is crucial for further exploration of the relationship between genotype, phenotype, and the function of a gene mutation.

In the study, the highest frequency of allele was exon 7 (33.25\%), followed by exons 11, 6, 12,3 , and 5 . More than $85 \%$ of mutations were detected by screening these six exons, suggesting there are hotspots for mutations in the PAH gene in PKU populations of China. Therefore, when screening, priority should be given to the sequencing of exons 6,7 , and 11 of the $P A H$ gene, which results in the detection of about $60 \%$ mutations. Including exons 3, 5, and 12 in the screening increases the detection to $85 \%$ of mutations. This strategy is timesaving, avoids laborious defects of whole coding region sequencing, and saves resources while improving the efficiency of diagnosis.

We identified five novel mutations that have not been indexed in relevant databases.

Our major challenge was to distinguish disease-associated mutations from SNPs. The c.463delC frameshift mutation results in the premature termination of $P A H$ gene translation and a truncated $\mathrm{PAH}$ enzyme. This mutation is generally considered the causative mutation of PKU. To investigate the remaining four missense mutations, we did a conservative analysis between species and then analyzed the mutation function by the PROVEAN prediction and PolyPhen-2 software. The results showed that these missense mutations sequence variation are highly conserved and are inclined to deleterious mutations. The pathogenic properties of the variation depends on the effects of PAH activity in in vitro expression. Some of the novel mutations were underlying the in vitro expression in our research. Our discovery of novel mutations enriches the human PAH gene mutation database.

Sanger sequencing can directly detect point mutations and small indels. MLPA was used to test for the presence of deletions or duplications in the PAH gene in a cohort of PKU patients in whom at least one mutation had remained undetected using standard diagnostic tools. After Sanger sequencing and MLPA, only one allele mutation was detected in five patients. We hypothesized that another allele mutation may be present in the regulatory region, exon intron, or distant polyadenylation sites or a SNP may be causing mRNA function changes and influencing the activity of the PAH protein function. Further studies are needed to identify the nature of mutation. 
The mutation detection rate was not $100 \%$ so prenatal genetic diagnosis was not possible for all families. STR indirect diagnosis can be used in order to complement $P A H$ gene sequence analysis and improve the detection rate. Goltsov et al. (1993) found STR polymorphic markers in intron 3, about $700 \mathrm{bp}$ from exon 3, to be associated with the PAH gene. The repeating unit ((TCTA)n) represents high polymorphism information and can be used in linkage analysis for prenatal diagnosis. The polymorphism information contents (PIC) $\mathrm{PAH}-1$ locus was between 0.680.73 in Chinese populations according to a previous study (Huang et al., 1995), but the single STR site cannot be used in the diagnosis for all families. We selected two additional polymorphic markers, $\mathrm{PAH}-26$ located at telomere $76 \mathrm{~kb}$ and $\mathrm{PAH}-32$ located at centromere $85 \mathrm{~kb}$, for linkage analysis, making the detection rate by STR $79.7 \%$. We used multiple fluorescent PCR combined with capillary electrophoresis for STR parting. Multiple PCR allows the amplification of three sites simultaneously, thereby avoiding diagnostic errors caused by sampling error. Additionally, STR parting is simple, accurate by capillary electrophoresis, and improves the working efficiency.

Although combined with multi polymorphic markers, the prenatal diagnosis rate can only achieve $80 \%$. In addition, multiple individuals from the family, especially the proband, are needed for STR linkage analysis, a condition that cannot always be met. Therefore, linkage analysis for prenatal diagnosis of PKU may not always be appropriate.

Hyperphenylalaninemia is a genetically heterogeneous disorder and can be caused by a deficiency of phenylalanine hydroxylase or by a tetrahydrobiopterin deficiency (BH4D). Linkage analysis based on the $P A H$ gene does not reliably detect this genetic heterogeneity and can lead to errors. Therefore detection of hyperphenylalaninemia by linkage analysis cannot be performed if hyperphenylalaninemia is not caused by a $P A H$ gene mutation.

Of the 118 fetuses from 112 families, we performed prenatal diagnosis through chorionic villus sampling, amniocentesis, or cordocentesis according to the gestational week. Prenatal diagnosis of the fetuses in the 107 core families was performed using $P A H$ gene sequencing and STR linkage analysis, resulting in an accurate diagnosis for all fetuses. For the five families in which the proband is deceased, the $P A H$ gene of the parents was sequenced and we confirmed that they were carriers. The deceased patients presumably had a $P A H$ gene mutation based on the clinical data and $P A H$ gene results of the parents. The five fetuses in non-core families were only genotyped by $P A H$ gene sequencing. In a single case, a dichorionic- diamniotic twin(DCDA), amniocentesis from two amniotic cavity was performed and genetic diagnosis showed that one fetus was a PKU patient while the other was only a carrier. The guardians chose selective feticide at 18 weeks after informed consent; the other fetus had a normal birth.

For the families in which probands are alive and had differential diagnosis based on $\mathrm{BH} 4$ loading test and $P A H$ gene sequencing, we recommend that prenatal genetic diagnosis should be performed using PAH gene sequencing and STR linkage analysis. While STR linkage analysis can provide prenatal diagnosis information, the accuracy of prenatal diagnosis is improved when combined with gene sequencing. When STR linkage analysis cannot provide information, gene sequencing might allow a prenatal diagnosis. STR analysis and $P A H$ gene sequencing complement each other and ensure the accuracy of the prenatal genetic diagnosis. When only one allele of the $P A H$ gene has a mutation in the proband, prenatal diagnosis must depend on the linkage analysis. If the proband cannot be tested, parents need to be screened for the $P A H$ gene. If the parents are identified as carriers, the prenatal diagnosis can be made by $P A H$ gene sequencing. Alternatively, if no mutation is detected in only one or both parents, the prenatal diagnosis cannot be offered.

The recent study of prenatal diagnosis of PKU has been infrequent. The majority of early 
studies are based on STR linkage analysis (Fang et al., 1992; Romano et al., 1994; Fan et al., 1999; Kohli et al., 2005; Fazeli et al., 2011), but there have been no cases of complete and large sample research. We investigated 118 cases of prenatal genetically diagnosed fetuses to test $P A H$ gene sequencing and STR linkage analysis methods. We confirmed that the methods are accurate and effective for PKU prenatal diagnosis. Furthermore, we propose a new PKU prenatal diagnosis strategy (Figure 3).

PKU is a remediable hereditary disease. Families requiring prenatal diagnosis must be fully informed, provide consent, and should have the doctor objectively explain the prenatal diagnosis results to them; the choice of how to proceed should be determined by the guardians. The prenatal diagnosis fetus should be performed in addition to neonatal screening after birth to verify the accuracy and reliability of the prenatal diagnosis and to allow the opportunity for early treatment if misdiagnosis has occurred.

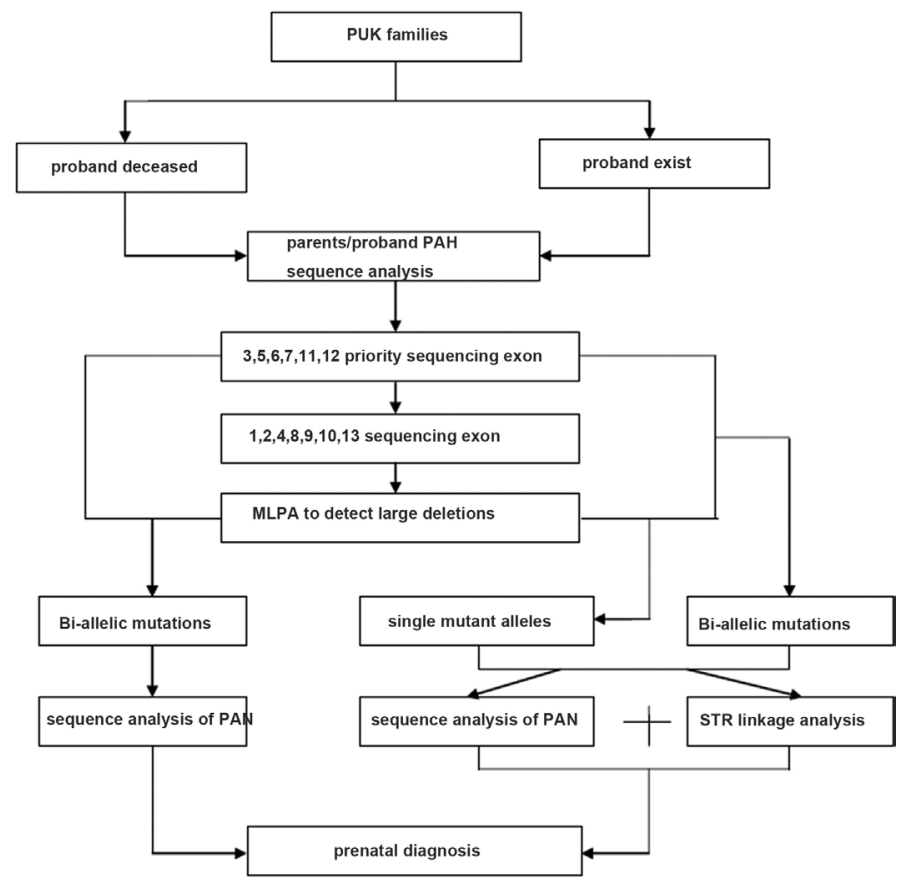

Figure 3. Strategy and flow chart for prenatal diagnosis of PKU.

\section{Conflicts of interest}

The authors declare no conflict of interest.

\section{ACKNOWLEDGMENTS}

We are grateful to the patients and their family members and to all participants in the study for their kind cooperation, especially Professor Zhao DeHua, Neonatal Screening Center, The Third Affiliated Hospital of Zhengzhou University. Research supported by the Henan Province Medical Science and Technique Foundation (Grant \#201303015 and \#201403021). 


\section{REFERENCES}

Blau N, van Spronsen FJ and Levy HL (2010). Phenylketonuria. Lancet 376: 1417-1427.

Fan GX, Qing LX, Jun Y and Mei Z (1999). Molecular studies and prenatal diagnosis of phenylketonuria in Chinese patients. Southeast Asian J. Trop. Med. Public Health 302: 63-65.

Fang B, Yuan L, Wang M, Huang S, et al. (1992). Detection of point mutations of the phenylalanine hydroxylase gene and prenatal diagnosis of phenylketonuria. Chin. Med. Sci. J. 7: 205-208.

Fazeli Z and Vallian S (2011). An artifact band frequently associated with variable number of tandem repeat marker at phenylalanine hydroxylase gene: application in carrier detection and prenatal diagnosis of phenylketonuria. Mol. Biol. Rep. 38: 3395-3399.

Goltsov AA, Eisensmith RC, Naughton ER, Jin L, et al. (1993). A single polymorphic STR system in the human phenylalanine hydroxylase gene permits rapid prenatal diagnosis and carrier screening for phenylketonuria. Hum. Mol. Genet. 2: 577-581.

Gu XF and Wang ZG (2004). Screening for phenylketonuria and congenital hypothyroidism in 5.8 million neonates in China. Zhonghua Yu Fang Yi Xue Za Zhi 38: 99-102.

Huang S, Fang B and Chu H (1995). Analysis of short tandem repeats polymorphism in the phenylalanine hydroxylase gene and its application to prenatal gene diagnosis of phenylketonuria. Zhonghua Yi Xue Za Zhi 75: 22-24.

Kohli S, Saxena R, Thomas E, Rao P, et al. (2005). Prenatal diagnosis of phenylketonuria. Indian J. Med. Res. 122: $400-403$.

Romano V, Dianzani I, Ponzone A, Zammarchi E, et al. (1994). Prenatal diagnosis by minisatellite analysis in Italian families with phenylketonuria. Prenat. Diagn. 14: 959-962.

Woo SL, Lidsky AS, Güttler F, Chandra T, et al. (1983). Cloned human phenylalanine hydroxylase gene allows prenatal diagnosis and carrier detection of classical phenylketonuria. Nature 306: 151-155.

Yao FX, Guo H, Han JJ, Meng Y, et al. (2007). The power of linkage analysis on PAH gene in prenatal gene diagnosis is improved with three additional short tandem repeat markers. Zhonghua Yi Xue Yi Chuan Xue Za Zhi 24: 382-386. 